\title{
A191 IMMUNE REGULATION BY PERIPHERAL TREGS INDUCED UPON HOMOTYPIC T CELL/T CELL INTERACTIONS
}

K Thümmler, J Leipe, A Ramming, I Prots, H S-Koops, A Skapenko Division of Rheumatology, Med. Poliklinik, University of Munich, Munich, Germany

\subsection{6/ard.2010.129676}

Autoimmune diseases like rheumatoid arthritis are characterised by persistently activated CD4 T cells, which circulate from the synovial tissues into the lymph nodes. Here, they encounter multiple contacts with bystander cells including resting CD4 $\mathrm{T}$ cells. We have recently shown that activated $T$ cells induce the proliferation and the production of cytokines with immunoregulatory potential from resting CD4 $T$ cells by homotypic $T$ cell interaction. Since the compromised function of regulatory $T$ cells results in the development of autoimmune diseases, we investigated the function of these $T$ cells resulting from the interaction of activated $T$ cells and resting CD4 $\mathrm{T}$ cells and the mechanism mediating this novel cellular interaction. Resting CD4 T cells were cocultured with fixed activated $T$ cells and analysed for their phenotype, cytokine secretion profile and immunoregulatory capacity.

$\mathrm{T}$ cells induced upon homotypic $\mathrm{T}$ cell interaction expressed CD25, reduced levels of CD127, transforming growth factor $\beta$, but no FOXP3. Of interest for the regulation of specific immune responses, the resulting cells strongly inhibited proliferation of CD25 negative T cells in a dose dependent manner as potently as natural occurring CD25 positive cells. Surprisingly, even polarised proinflammatory effector cells (eg, T-helper 1 (Th1) or Th17 cells) induced Tregs from memory CD4 $\mathrm{T}$ cells. The inhibitory effect was partly contact dependent, partly dependent on cytokines and could be abrogated by high amounts of exogenous interleukin 2 (IL-2). In vivo, Tregs resulting from the interaction of resting DO11.10 CD4 $\mathrm{T}$ cells and activated $\mathrm{T}$ cells from Balb/c mice suppressed the expansion of ovalbumin-specific T cells upon antigen challenge in the DO11.10 transfer model. Blocking adhesion receptor/counterreceptor interaction with $\mathrm{mAbs}$ to particular ligands revealed that the generation of regulatory $\mathrm{T}$ cells by homotypic $\mathrm{T}$ cell 
contact is both, anchored and tuned through interactions between leucocyte function-associated antigen 1 (LFA-1) and its ligands, ICAM-1, -2 and -3 . While blocking of LFA-1 prevented the generation of Tregs, $m A$ bs to ICAM-1 diminished proliferation of the responder cells and neutralisation of ICAM-3 reduced IL-4 secretion.
Our data indicate a novel negative feedback mechanism via bystander immune modulation, where activated proinflammatory effector $T$ cells induce the generation of Tregs from resting $T$ cells. The data, thus, suggest that homotypic $T$ cell interactions represent a physiological means to counteract sustained inflammation. 